1 Hacettepe Journal of Mathematics and Statistics

$\bigcap$ Volume 45 (1) (2016), 43-48

\title{
On a conjecture of double inequality for the tangent function
}

\author{
Mansour Mahmoud *
}

\begin{abstract}
In this paper, we prove the conjecture posed by J.-L. Zhao, Q.-M. Luo, B.-N. Guo and F. Qi (Remarks on inequalities for the tangent function, Hacettepe J. Math. Stat., 41, no. 4, 499-506, 2012) about a sharp double inequality of the tangent function, which is a generalization of the Becker-Stark inequality. Also, the new double inequality is compared with the double inequality presented in [3]
\end{abstract}

Keywords: Tangent function, L'Hôpital's rule, inequality, best constants, concavity.

2000 AMS Classification: 26D05, 26D15.

Received : 17.04.2014 Accepted : 29.01.2015 Doi : 10.15672/HJMS.20164512486

\section{Introduction}

In 1955, Stečkin [14] obtained the following one-side inequality for the tangent function

$$
\frac{\tan x}{x}>\frac{4 / \pi}{\pi-2 x}, \quad 0<x<\pi / 2
$$

where the constant $4 / \pi$ is the best possible.

Later in 1978, Becker and Stark [2] presented the following double inequality

$$
\frac{8}{\pi^{2}-4 x^{2}}<\frac{\tan x}{x}<\frac{\pi^{2}}{\pi^{2}-4 x^{2}}, \quad 0<x<\pi / 2
$$

which is a generalization of the Stečkin's inequality (1.1).

${ }^{*}$ King Abdulaziz University, Faculty of Science, Mathematics Department, P. O. Box 80203, Jeddah 21589, Saudi Arabia.

Email: mansour@mans.edu.eg

Permanent address: Mansour Mahmoud, Department of Mathematics, Faculty of Science, Mansoura University, Mansoura 35516, Egypt. 
In 2003, C.-P. Chen and F. Qi [3] established a double inequality for remainder $r_{n}(x)=$ $\tan x-S_{n}(x)$, where $S_{n}(x)$ is the $n^{t h}$ partial sum of the power series of $\tan x$. Their double inequality can be reformulated as [16]:

1.1. Theorem. For $0<x<\pi / 2$ and $n \in \mathbb{N}$, we have

$$
\frac{2^{2(n+1)}\left(2^{2(n+1)}-1\right)\left|B_{2 n+2}\right|}{(2 n+2) !} x^{2 n} \tan x<\tan x-S_{n}(x)<\left(\frac{2}{\pi}\right)^{2 n} x^{2 n} \tan x,
$$

where

$$
S_{n}(x)=\sum_{i=1}^{n} \frac{2^{2 i}\left(2^{2 i}-1\right)\left|B_{i}\right|}{(2 i) !} x^{2 i-1}
$$

and $B_{j}$ 's are the Bernoulli numbers.

The inequality (1.3) for $n=1$ and $0<x<\frac{3}{2} \sqrt{\frac{5\left(\pi^{2}-8\right)}{38}}$ will give us a refinement of the left-hand side of the Becker-Stark inequality (1.2). Also, the inequality (1.3) for $n=2$ is better than the Djokovic inequality [8]

$$
x+\frac{1}{3} x^{3}<\tan x<x+\frac{4}{9} x^{3}, \quad 0<x<\pi / 6 .
$$

In 2010, Zhu and Hua [17] established the following general refinement of the BeckerStark inequality

1.2. Theorem. Let $0<x<\pi / 2$ and a natural number $n \geq 0$. Then

$$
\frac{P_{2 n}(x)+\alpha_{n} x^{2 n+2}}{\pi^{2}-4 x^{2}}<\frac{\tan x}{x}<\frac{P_{2 n}(x)+\beta_{n} x^{2 n+2}}{\pi^{2}-4 x^{2}},
$$

where $P_{2 n}(x)=\sum_{i=0}^{n} a_{i} x^{2 i}$ and

$$
a_{i}=\pi^{2}\left|B_{2 i+2}\right| \frac{2^{2(i+1)}\left(2^{2(i+1)}-1\right)}{(2 i+2) !}-4\left|B_{2 i}\right| \frac{2^{2 i}\left(2^{2 i}-1\right)}{(2 i) !}, \quad i=0,1,2, \ldots .
$$

Furthermore, $\alpha_{n}=\frac{8-P_{2 n}(\pi / 2)}{(\pi / 2)^{2 n+2}}$ and $\beta_{n}=\alpha_{n+1}$ are the best constants in (1.5).

In 2012, Zhao, Luo, Guo and Qi [16] showed that the double inequalities (1.3) and (1.5) are not included in each other, reorganized the proof of (1.3) by using the usual definition of Bernoulli numbers and corrected some errors on [12]. Moreover, they propose a sharp double inequality as a conjecture. In this paper we will prove this conjecture. Further interesting generalizations and applications about inequalities of the tangent function can be found in [4]-[6], [9], [10], [15], [18]-[20] and the references therein.

In our present investigation, we will apply the following monotone form of L'Hôpital's rule [1] (see also, [7], [11], [13] ).

1.3. Theorem. Let $-\infty<a<b<\infty$, and let $f, g:[a, b] \rightarrow \mathbb{R}$ be continuous on $[a, b]$, differentiable on $(a, b)$. Let $g^{\prime}(x) \neq 0$ on $(a, b)$. If $f^{\prime}(x) / g^{\prime}(x)$ is increasing (decreasing) on $(a, b)$, then so are

$$
\frac{f(x)-f(a)}{g(x)-g(a)} \quad \text { and } \quad \frac{f(x)-f(b)}{g(x)-g(b)} .
$$

If $f^{\prime}(x) / g^{\prime}(x)$ is strictly monotone, then the monotonicity in the conclusion is also strict. 


\section{Main Results.}

Consider the following two functions for $n \in \mathbb{N}$ and $x \in\left(0, \frac{\pi}{2}\right)$

$$
M_{n}(x)=\frac{\tan x}{S_{n}(x)}\left[1-\left(\frac{2 x}{\pi}\right)^{2 n}\right]
$$

and

$$
h_{n}(x)=\frac{\tan x-S_{n}(x)}{x^{2 n} \tan x} .
$$

Then

$$
\frac{1}{M_{n}(x)}=\frac{1-x^{2 n} h_{n}(x)}{1-(2 / \pi)^{2 n} x^{2 n}}
$$

Let

$$
f_{n}(x)= \begin{cases}1-x^{2 n} h_{n}(x) & 0<x \leq \pi / 2, \\ 1 & x=0\end{cases}
$$

and

$$
g_{n}(x)=1-(2 / \pi)^{2 n} x^{2 n}, \quad 0 \leq x \leq \pi / 2 .
$$

The functions $f_{n}, g_{n}:\left[0, \frac{\pi}{2}\right] \rightarrow \mathbb{R}$ are continuous on $\left[0, \frac{\pi}{2}\right]$, differentiable on $\left(0, \frac{\pi}{2}\right)$. Also, $g_{n}^{\prime}(x)=-2 n(2 / \pi)^{2 n} x^{2 n-1} \neq 0$ on $\left(0, \frac{\pi}{2}\right)$. Now, consider the function

$$
G_{n}(x)=\frac{f_{n}^{\prime}(x)}{g_{n}^{\prime}(x)}=(\pi / 2)^{2 n}\left[\frac{x}{2 n} h_{n}^{\prime}(x)+h_{n}(x)\right],
$$

then

$$
G_{n}^{\prime}(x)=\frac{(\pi / 2)^{2 n}}{2 n}\left[(2 n+1) h_{n}^{\prime}(x)+x h_{n}^{\prime \prime}(x)\right] .
$$

But the function $h_{n}(x)$ is absolutely monotonic on $\left(0, \frac{\pi}{2}\right)$ [16], that is

$$
\left(h_{n}(x)\right)^{(i)} \geq 0, \quad \forall i \in \mathbb{N} ; x \in\left(0, \frac{\pi}{2}\right) .
$$

Then

$$
G_{n}^{\prime}(x)>0, \quad x \in\left(0, \frac{\pi}{2}\right)
$$

and hence the function $G_{n}=\frac{f_{n}^{\prime}}{g_{n}^{\prime}}$ is increasing function on $\left(0, \frac{\pi}{2}\right)$. Using Theorem 1.3, we get that

$$
\frac{f_{n}(x)-f_{n}(\pi / 2)}{g_{n}(x)-g_{n}(\pi / 2)}
$$

is also increasing on $\left(0, \frac{\pi}{2}\right)$. But $f_{n}(\pi / 2)=g_{n}(\pi / 2)=0$ and hence $\frac{1}{M_{n}(x)}=\frac{f_{n}(x)}{g_{n}(x)}$ is increasing on $\left(0, \frac{\pi}{2}\right)$. Then $M_{n}(x)$ is decreasing on $\left(0, \frac{\pi}{2}\right)$. Then

$$
\lim _{x \rightarrow \frac{\pi \frac{\pi}{2}^{-}}{}} M_{n}(x)<M_{n}(x)<\lim _{x \rightarrow 0^{+}} M_{n}(x) .
$$

Using

$$
\lim _{x \rightarrow 0^{+}} M_{n}(x)=\lim _{x \rightarrow 0^{+}} \frac{\tan x}{S_{n}(x)}=\lim _{x \rightarrow 0^{+}} \frac{\tan x / x}{1+\sum_{i=2}^{n} \frac{2^{2 i}\left(2^{2 i}-1\right)\left|B_{i}\right|}{(2 i) !} x^{2(i-1)}}=1
$$

and

$$
\lim _{x \rightarrow \frac{\pi}{2}^{-}} M_{n}(x)=\frac{1}{S_{n}(\pi / 2)} \lim _{x \rightarrow \frac{\pi^{-}}{2}} \frac{1-\left(\frac{2 x}{\pi}\right)^{2 n}}{\cot x}=\frac{4 n / \pi}{S_{n}(\pi / 2)},
$$

we get the following result 
2.1. Theorem ([16], Conjecture 1). For $0<x<\pi / 2$ and $n \in \mathbb{N}$, we have

$$
\frac{4 n / \pi}{S_{n}(\pi / 2)}<\frac{\tan x}{S_{n}(x)}\left[1-\left(\frac{2 x}{\pi}\right)^{2 n}\right]<1 .
$$

Furthermore, 1 and $\frac{4 n / \pi}{S_{n}(\pi / 2)}$ are the best possible constants in (2.4).

2.2. Remark. If we set $n=1$ in the inequality (2.4), then we obtain the inequality (1.2) and hence the inequality (2.4) is an extension of Becker-Stark inequality (1.2).

Now we will study the concavity of the function $M_{n}(x)$. Let us recall that, a function $\varphi$ is concave if every chord lies below the graph of $\varphi$. Let $y_{n}(x)$ be the line segment with the endpoints $(0,1)$ and $\left(\frac{\pi}{2}, \frac{4 n / \pi}{S_{n}(\pi / 2)}\right)$. Then

$$
y_{n}(x)=\left[\frac{4 n / \pi}{S_{n}(\pi / 2)}-1\right] \frac{2 x}{\pi}+1, \quad 0 \leq x \leq \pi / 2
$$

and let

$$
H_{n}(x)= \begin{cases}1 & x=0 \\ M_{n}(x) & 0<x<\pi / 2 \\ \frac{4 n / \pi}{S_{n}(\pi / 2)} & x=\pi / 2\end{cases}
$$

The functions $H_{n}, y_{n}:\left[0, \frac{\pi}{2}\right] \rightarrow \mathbb{R}$ are continuous on $\left[0, \frac{\pi}{2}\right]$, differentiable on $\left(0, \frac{\pi}{2}\right)$. Also, $y_{n}^{\prime}(x)=\left[\frac{4 n / \pi}{S_{n}(\pi / 2)}-1\right] \neq 0 \forall x$. Now consider the function

$$
T_{n}(x)=\frac{H_{n}^{\prime}(x)}{y_{n}^{\prime}(x)}=\frac{\pi}{2\left(\frac{4 n / \pi}{S_{n}(\pi / 2)}-1\right)} H_{n}^{\prime}(x) .
$$

If we assume that $H_{n}^{\prime \prime}(x)>0$, then we get $T_{n}(x)$ is decreasing function. Using Theorem 1.3 , we get that

$$
F(x)=\frac{H_{n}(x)-H_{n}(0)}{y_{n}(x)-y_{n}(0)}=\frac{H_{n}(x)-1}{y_{n}(x)-1}
$$

is also decreasing function on $\left(0, \frac{\pi}{2}\right)$. But

$$
\lim _{x \rightarrow 0^{+}} F(x)=\lim _{x \rightarrow 0^{+}} \frac{\pi}{2\left(\frac{4 n / \pi}{S_{n}(\pi / 2)}-1\right)} H_{n}^{\prime}(x)=0
$$

and

$$
\lim _{x \rightarrow \frac{\pi}{2}^{-}} F(x)=\frac{H_{n}(\pi / 2)-1}{y_{n}(\pi / 2)-1}=1,
$$

which is a contradiction since $F(x)$ is decreasing. Then we get the following result

2.3. Lemma ([16], Conjecture 1). For $0<x<\pi / 2$ and $n \in \mathbb{N}$, the function

$$
M_{n}(x)=\frac{\tan x}{S_{n}(x)}\left[1-\left(\frac{2 x}{\pi}\right)^{2 n}\right]
$$

is concave. 


\section{Comparison of Theorems 1.1 and 2.1 .}

The inequalities in Theorems 1.1 and 1.2 are not included in each other [16]. Now, we will compare the Theorems 1.1 and 2.1 .

The inequality (1.3) can be rewritten in the form

$$
\frac{1}{1-\left(\frac{2^{2(n+1)}\left(2^{2(n+1)}-1\right)\left|B_{2(n+1)}\right|}{(2 n+2) !}\right) x^{2 n}}<\frac{\tan x}{S_{n}(x)}<\frac{1}{1-\left(\frac{2}{\pi}\right)^{2 n} x^{2 n}}
$$

and the inequality (2.4) can be rewritten in the form

$$
\frac{4 n}{\pi S_{n}(\pi / 2)\left(1-\left(\frac{2}{\pi}\right)^{2 n} x^{2 n}\right)}<\frac{\tan x}{S_{n}(x)}<\frac{1}{1-\left(\frac{2}{\pi}\right)^{2 n} x^{2 n}} .
$$

So, the two inequalities (3.1) and (3.2) have the same upper bound. To compare the lower bounds of (3.1) and (3.2), take $n=1,2,3$ in the left-hand side of (3.1), to obtain

$$
\begin{array}{ll}
n=1 & L_{1}(x)=\frac{1}{1-\frac{x^{2}}{3}} \\
n=2 & L_{2}(x)=\frac{1}{1-\frac{2 x^{4}}{15}} \\
n=3 & L_{3}(x)=\frac{1}{1-\frac{17 x^{6}}{315}}
\end{array}
$$

and set $n=1,2,3$ in the left-hand side of (3.2), to get

$$
\begin{array}{ll}
n=1 & K_{1}(x)=\frac{8}{\pi^{2}\left(1-\frac{4 x^{2}}{\pi^{2}}\right)} \\
n=2 & K_{2}(x)=\frac{8}{\pi\left(\pi / 2+\pi^{3} / 24\right)\left(1-\frac{16 x^{4}}{\pi^{4}}\right)} \\
n=3 & K_{3}(x)=\frac{12}{\pi\left(\pi / 2+\pi^{3} / 24+\pi^{5} / 240\right)\left(1-\frac{64 x^{6}}{\pi^{6}}\right)} .
\end{array}
$$

Then

$$
\begin{aligned}
& L_{1}(x)>K_{1}(x) \quad \text { if } 0<x<\frac{1}{2} \sqrt{3\left(\pi^{2}-8\right)} \\
& L_{1}(x)<K_{1}(x) \quad \text { if } \frac{1}{2} \sqrt{3\left(\pi^{2}-8\right)}<x<\pi / 2 \\
& L_{2}(x)>K_{2}(x) \quad \text { if } 0<x<\frac{1}{2} \sqrt[4]{\frac{5\left(-192 \pi^{2}+12 \pi^{4}+\pi^{6}\right)}{3\left(20-\pi^{2}\right)}} \\
& L_{2}(x)<K_{2}(x) \quad \text { if } \frac{1}{2} \sqrt[4]{\frac{5\left(-192 \pi^{2}+12 \pi^{4}+\pi^{6}\right)}{3\left(20-\pi^{2}\right)}}<x<\pi / 2 \\
& L_{3}(x)>K_{3}(x) \quad \text { if } 0<x<\frac{1}{2} \sqrt[6]{\frac{7\left(-2880 \pi^{4}+120 \pi^{6}+10 \pi^{8}+\pi^{10}\right)}{10\left(84+7 \pi^{2}-\pi^{4}\right)}} \\
& L_{3}(x)<K_{3}(x) \quad \text { if } \frac{1}{2} \sqrt[6]{\frac{7\left(-2880 \pi^{4}+120 \pi^{6}+10 \pi^{8}+\pi^{10}\right)}{10\left(84+7 \pi^{2}-\pi^{4}\right)}}<x<\pi / 2 \text {. }
\end{aligned}
$$

Hence, the lower bounds of (3.1) and (3.2) are not included in each other. Also, we can conclude that inequality (3.1) is better than inequality (3.2) near the origin and that inequality (3.2) is better than inequality (3.1) near $\pi / 2$.

\section{References}

[1] Anderson, G. D., Vamanamurthy, M. K. and Vuorinen, M. Monotonicity of some functions in Calculus.

(Available online at https://www.math.auckland.ac.nz/Research/Reports/Series/538.pdf).

[2] Becker, M. and Stark, E. L. On hierarchy of polynomial inequalities for $\tan (x)$, Univ. Beograd, Publ. Elektrotehn. Fak. Ser. Mat. Fiz. No. 602-633, 133-138, 1978.

[3] Chen, C.-P. and Qi, F. A double inequality for remainder of power series of tangent function, Tamkang Journal of Mathematics 34 (4), 351-355, 2003.

[4] Chen, C.-P. and Cheung, W.-S. Sharp Cusa and Becker-Stark inequalities. Journal of Inequalities and Applications 2011 2011:136. 
[5] Debnath, L., Mortici, C. and Zhu L. Refinements of Jordan-Stečkin and Becker-Stark inequalities, Results Math. DOI: 10.1007/s00025-014- 0405-3

[6] Ge, H.-F. New sharp bounds for the Bernoulli numbers and refinement of Becker-Stark inequalities, Journal of Applied Mathematics, Article ID 137507, 7 pages, 2012.

[7] Hardy, G. H., Littlewood, J. E. and Pólya, G. Inequalities, Cambridge Univ. Press, 1959.

[8] Kuang, J.-Ch. Chángyòng Bùděngshì(Applied inequalities), $3^{\text {rd }}$ ed., Shāndōng Kēxué Jìshù Chūbăn Shè (Shandong Science and Technology Press), Jinan City, Shandong Province, China, 2004 (Chinese).

[9] Mortici, C. The natural approach of Wilker-Cusa-Huygens inequalities, Math. Inequal. Appl. 14, no. 3, 535-541, 2011.

[10] Mortici, C. A subtly analysis of Wilker inequality, Applied Mathematics and Computation, 231, 516-520, 2014.

[11] Pinelis, I. L'Hospital rules for monotonicity and the Wilker-Anglesio inequality, Amer. Math. Monthly 111 (10), 905-909, 2004.

[12] Qi, F., Niu, D.-W. and Guo, B.-N. Refinements, generalizations and applications of Jordan's inequality and related problems, Journal of Inequalities and Applications, Article ID 271923, 52 pages, 2009.

[13] Sándor, J. Sharp Cusa-Huygens and related inequalities, Notes on Number Theory and Discrete Mathematics 19 (1), 50-54, 2013.

[14] Stečkin, S. B. Some remarks on trigonometric polynomials (Russian). Uspehi Mat. Nauk (N.S.) 10, No. 1 (63), 159-166, 1955.

[15] Sun, Z. J. and Zhu, L. Simple proofs of the Cusa-Huygens-type and Becker-Stark-type inequakities, Journal of Mathematical Inequalities, 7 (4), 563-567, 2013.

[16] Zhao, J.-L., Luo, Q.-M., Guo, B.-N. and Qi, F. Remarks on inequalities for the tangent function, Hacettepe J. Math. Stat., 41 (4), 499-506, 2012.

[17] Zhu, L. and Hua, J.-K. Sharpening the Becker-Stark inequalities, Journal of Inequalities and Applications, Article ID 931275, 2010.

[18] Zhu, L. Sharp Becker-Stark-type inequalities for Bessel functions, Journal of Inequalities and Applications, Vol. 2010, Article ID 838740, 4 pages.

[19] Zhu, L. A refinement of the Becker-Stark inequalities, Mat. Zametki, 93 (3), 401-406, 2013.

[20] Zhu, L. and Mortici, C. Inequalities Becker-Stark at extreme points, arXiv:1312.6276 [math.CA]. 